\title{
Optimization of demolding temperature for throughput improvement of nanoimprint lithography
}

\author{
T. Leveder ${ }^{\mathrm{a}, *}$, S. Landis ${ }^{\mathrm{a}}$, L. Davoust $^{\mathrm{b}}$, N. Chaix ${ }^{\mathrm{c}}$ \\ ${ }^{a}$ CEA Leti-Minatec 17 rue des martyrs, 38054 Grenoble, France \\ ${ }^{\mathrm{b}}$ LEGIIENSHMG, 1025 rue de la piscine Dom. Univ. BP 53, 38041 Grenoble, France \\ ${ }^{\mathrm{c}}$ CNRS-LTM, 17 rue des martyrs, 38054 Cedex 9, Grenoble, France
}

Annealing effects onto the reflow of imprinted resist patterns have been investigated on $250 \mathrm{~nm}$ dense line arrays printed with standard hot embossing lithography and thermoplastic polymer. Atomic force microscopy measurements were performed to point out the annealing temperature and time effects, respectively. The reflow velocity with respect to annealing temperature has been determined. Its variation is ascribed to both resist dynamic viscosity and surface free energy. Our approach demonstrated that imprint cycle time could be significantly reduced by saving cooling down time.

Keywords: Nanoimprint lithography; Patterns reflow; Demolding modelisation; Throughput optimization

\section{Introduction}

The international technology roadmap for semiconductor (ITRS) trends predicts constant diminution of the features width and improvement of their quality. Whereas wavelength downsizing or immersion techniques are proposed to improve capabilities of conventional projection lithographies, other approaches are proposed for next generation lithographies (NGL). Nanoimprint lithographies are emerging as the long-awaited disruptive technologies. Among them, hot embossing lithography appears as the most flexible and low-cost technology. Contrary to UV nanoimprint lithography (UV-NIL), hot embossing lithography is free from a lot of material issues. The two mains advantages are that resist do not need to be UV curable and quartz stamps are not required. De facto, thermal nanoimprint lithography has achieved great results since Chou et al. has demonstrated imprint feasibility in 1995

\footnotetext{
${ }^{*}$ Corresponding author.

E-mail addresses: tanguy.leveder@cea.fr (T. Leveder), slandis@cea.fr (S. Landis).
}

[1]. Molecular-scale resolution has been reached recently [2], and no other technology allows getting $2 \mathrm{~nm}$ features at such a low cost. Moreover, nanoimprint is for now the only way to build 3D features in one step [3].

Despite of tremendous advances since mid nineties, NIL has to prove to be a viable industrial alternative to extreme ultraviolet (EUV) lithography for $32 \mathrm{~nm}$ device generation and beyond. One of the main industrial issues is to reach a critical throughput to be competitive with current lithography systems. From a manufacturing point of view, considering equipment costs and production rates, the process cycle times should be as short as possible [4]. It is also desirable to improve stamp durability by getting the smoothest possible temperature cycle.

In this paper, we will focus on a way to perform a quasi constant temperature imprint. By saving heating up time and cooling down times which are used to set the printed resist above and below the glass transition temperature $\left(T_{\mathrm{g}}\right)$, it is possible to significantly reduce cycle time on every imprint. Our approach will give a set of rules, which allows choosing a process temperature giving a good balance between the required pattern quality and a process time low enough. 
The main issue of quasi constant temperature imprint is to keep a good pattern quality, despite of high temperature demolding. In order to study pattern evolution after such a demolding, we experimentally reproduce this process. Usual imprint steps to get good quality patterns were performed - heating, printing, cooling down - and then successive annealing were carried out to characterize reflowing shape.

First, we will present our experimental set-up. Then, impact of annealing temperature and time onto pattern quality will be described. Finally, we will focus on the definition of demolding quality criteria.

\section{Experimental section}

\subsection{Printing conditions}

Printing processes have been performed on $\mathrm{EVG}^{\circledR}$ $520 \mathrm{HE}$ system [5] and were performed under vacuum by pumping the chamber down to $5 \times 10^{-4}$ bar.

The $200 \mathrm{~mm}$ silicon stamp used for this paper was manufactured with DUV optical lithography and reactive ion etching. A $250 \mathrm{~nm}$ dense line array, $180 \mathrm{~nm}$ deep, have been printed into $100 \mathrm{~nm}$ thick $\mathrm{mr}-\mathrm{I}$ 7010E resist from Micro Resist Technology. The resist film was thick enough to ensure a complete filling of the stamp. It has been printed at $110^{\circ} \mathrm{C}\left(T_{\mathrm{g}}+50^{\circ} \mathrm{C}\right)$ under $40 \mathrm{kN}$ for $2 \mathrm{~min}$. Then substrates were cooled down before demolding in order to prevent from any resist reflow.

\subsection{Metrology}

Our approach consists in performing first classical imprint lithography (Fig. 1a) and then heating resists pattern obtained above the glass transition temperature (Fig. 1b). The initial resist features are $250 \mathrm{~nm}$ dense lines, $180 \mathrm{~nm}$ high with sidewall angle larger than $85^{\circ}$.
In order to look at resist patterns reflow above $T_{\mathrm{g}}$ in great details, samples were annealed for different times. After a short baking, samples are quenched to ambient temperature $\left(20^{\circ} \mathrm{C}\right)$. Then, frozen shapes of patterns were measured with an atomic force microscopy set-up.

Accurate measurements of the reflowing shape may be difficult, even if measurement is performed onto frozen states. Indeed, for slight resist reflowing (small baking times) the patterns were still vertical with sidewall angles larger than $70^{\circ}$. Standard conic shape AFM tips were therefore inappropriate for such characterizations. For small annealing times flared shape tips onto Veeco AFM $\mathrm{X} 3 \mathrm{D}$ were then used. Such tips can access re-entrant patterns but not acute angles.

Thus, the beginning of reflow is measurable with flared tips (Fig. 2a), whereas the end is measurable with conic tips (Fig. 2b). Consequently, we could access accurate measurements of the whole evolution of a reflowing grating as a function of time. Those measurements have been performed on the same resist for four different temperatures: 80,90 , 100 and $110^{\circ} \mathrm{C}$. That is to say from 20 to $50{ }^{\circ} \mathrm{C}$ above the glass transition temperature of the $\mathrm{mr}-\mathrm{I} 7000 \mathrm{E}$ resist.

\subsection{Experimental results}

\subsubsection{Influence of annealing time}

Quantitative characterization of resist patterns is needed to define a process window for a stamp-substrate demolding at printing temperature higher than glass transition temperature. In this paper, we will look at pattern height modification with respect to annealing time for different temperatures.

Fig. 3 shows the cross-sectional profile evolution for an annealing time varying from 1 to $500 \mathrm{~s}$. Printed resist patterns were annealed at $80^{\circ} \mathrm{C}\left(T_{\mathrm{g}}+20^{\circ} \mathrm{C}\right)$. We used flared tip as long as the side angle was larger than $77^{\circ}$. Then, for smoother profiles - i.e. annealing time larger than a
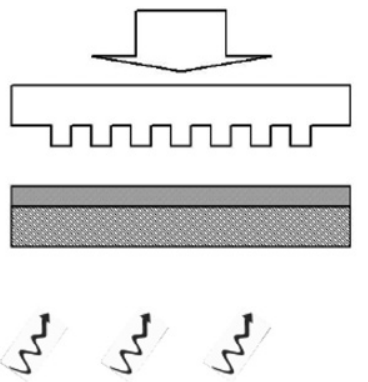

b

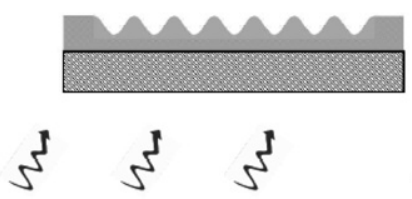

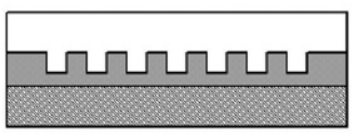
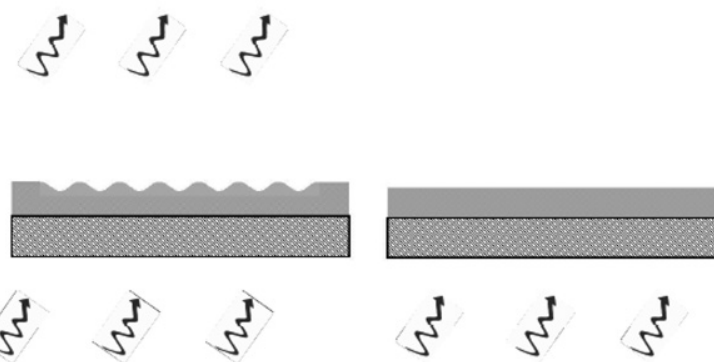

Fig. 1. (a) Imprint at temperature $(T)$ above $T_{\mathrm{g}}$ and demolding scheme $\left(T<T_{\mathrm{g}}\right)$. (b) Post demolding baking step and lines reflowing at temperature above $T_{\mathrm{g}}$. 


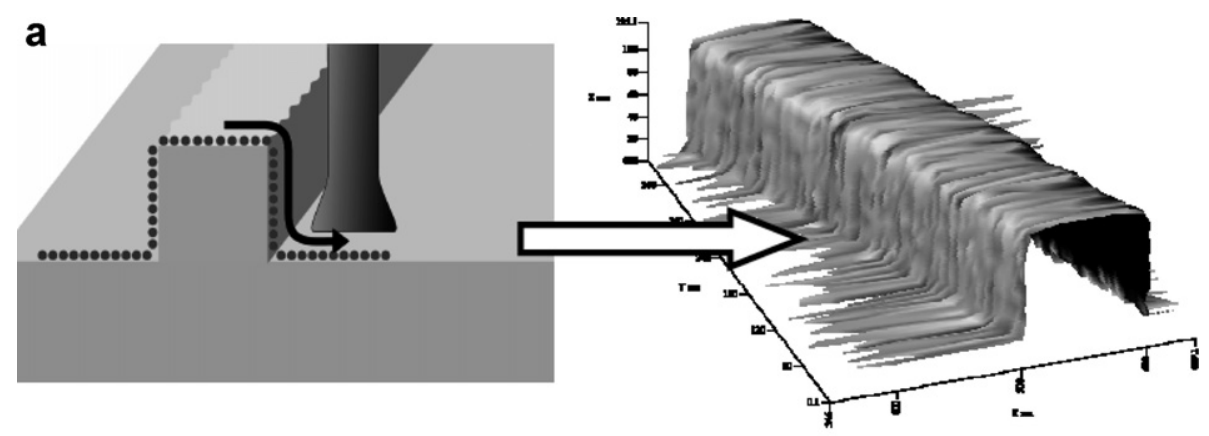

b

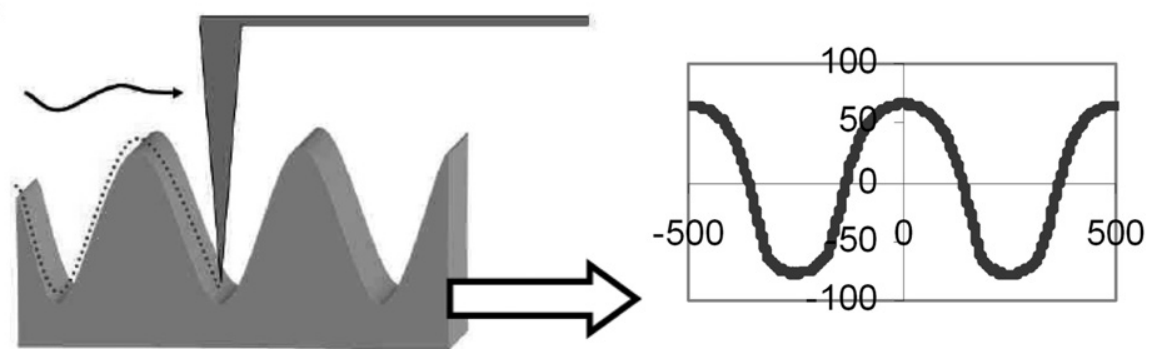

Fig. 2. AFM measurements (a) with flare tips for steep resist profiles and (b) with conic tips for smother resist profiles.

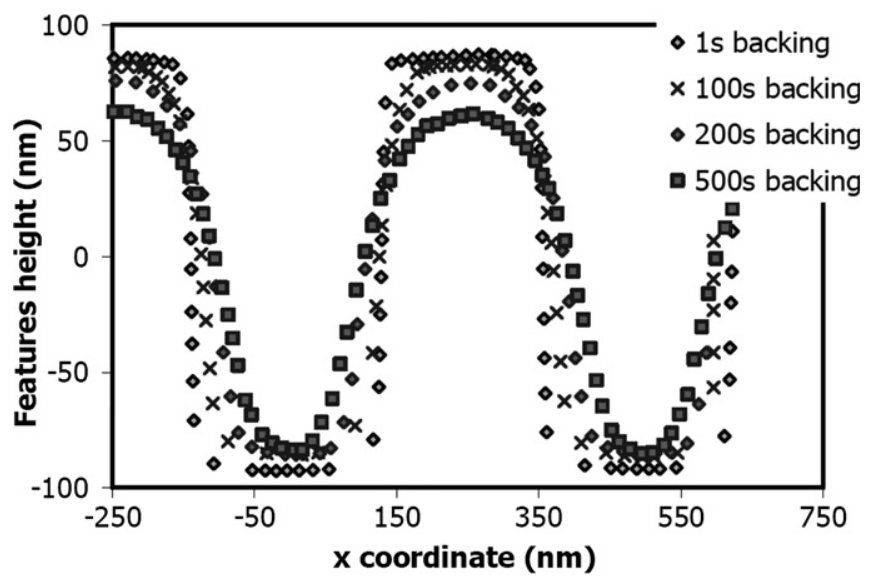

Fig. 3. AFM cross-sectional profiles of annealed resist patterns. The annealing temperature and time were $80^{\circ} \mathrm{C}$ and from $1 \mathrm{~s}$ to $500 \mathrm{~s}$, respectively.

$100 \mathrm{~s}$ - conic tips are sharp enough to explore the bottom of trenches.

Increasing annealing time, the cross-sectional profiles become more rounded and pattern height decreased. This resist flow is clearly related to the surface free energy which tends to minimize interfacial surface between resist and air. This characterization may be useful to optimize the demolding at temperature above $T_{\mathrm{g}}$. By considering that less than $3 \%$ of height variation, for example, is acceptable during the demolding, the maximum cooling time can be determined from Fig. 3. It implies that demolding at $T_{\mathrm{g}}+20^{\circ} \mathrm{C}$ and then cooling down below $T_{\mathrm{g}}$ should be performed in less than $100 \mathrm{~s}$.

\subsubsection{Influence of annealing temperature}

Fig. 3 shows that resist reflow was very sensitive to annealing time. Similar characterizations were carried out for different annealing temperatures ranging from 80 to $110{ }^{\circ} \mathrm{C}$. The annealing time was fixed to $30 \mathrm{~s}$.

Similarly to results shown in Fig. 3, the cross-sectional profiles became more rounded after an annealing above $T_{\mathrm{g}}$. In addition, the evolution of resist patterns is larger for higher annealing temperature than for longer annealing time. Indeed, pattern shape achieved after an annealing at $80^{\circ} \mathrm{C}$ for $500 \mathrm{~s}$ is very close to the one achieved after annealing the resist at $110^{\circ} \mathrm{C}$ for $30 \mathrm{~s}$. As reflow is very sensitive to temperature, our results indicate that polymer dynamic viscosity plays a key role.

\section{Results analysis}

\subsection{Process window determination}

From Figs. 3 and 4, the evolution of the pattern height, i.e. the difference between the highest and lowest point in the cross-sectional profiles, have been determined for annealing temperature between $80^{\circ} \mathrm{C}$ and $110^{\circ} \mathrm{C}$ and annealing time up to $500 \mathrm{~s}$ (Fig. 5). Each experimental point corresponds to an average value over 20 scan lines onto three printed resist lines. The deviation from the mean height value is lower than $2 \%$, so that we could consider that measurements are very reliable. Fig. 5 clearly shows that annealing temperature has a much larger effect onto the resist reflow than annealing time. The linear-log scale also reveals that experimental evolution of the resist feature height may be well fitted with an exponential law such as $h(t)=h_{0} \mathrm{e}^{-(t / \tau)}$,

$h_{0}$ the initial resist height of the pattern and $\tau$ a characteristic time of the resist reflow. The characteristic time $\tau$ is ranging from $2490 \mathrm{~s}$ at $80^{\circ} \mathrm{C}$ down to $40 \mathrm{~s}$ at $110^{\circ} \mathrm{C}$. 


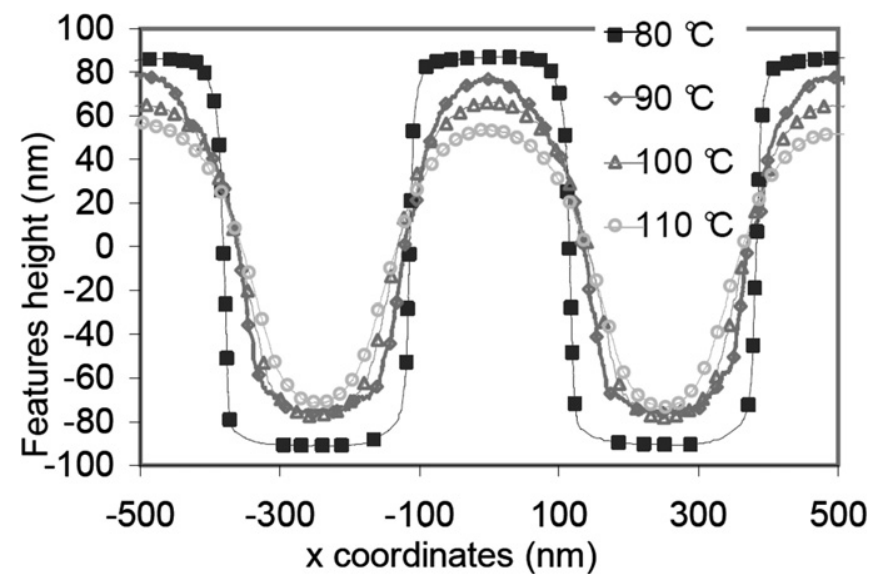

Fig. 4. AFM cross-sectional profiles of annealed resist patterns. The annealing time was $30 \mathrm{~s}$ and annealing temperatures were ranging from $T_{\mathrm{g}}+20^{\circ} \mathrm{C}\left(80^{\circ} \mathrm{C}\right)$ to $T_{\mathrm{g}}+50^{\circ} \mathrm{C}\left(110^{\circ} \mathrm{C}\right)$.

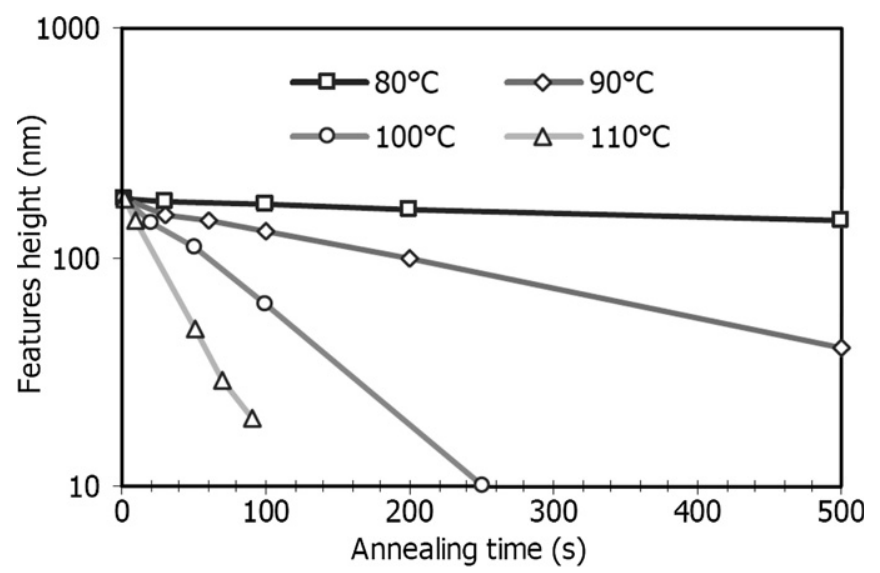

Fig. 5. Feature height variation with respect to annealing time. A post demolding baking at $80,90,110$ and $110^{\circ} \mathrm{C}$ were performed onto the printed lines.

Theoretical works are underway to clearly establish a relationship between $\tau$ and the flow properties of the polymer.

Even if the theoretical background of such flow behavior is not yet fully understood, it is possible from Fig. 5 to define a demolding process window. It defines the maximum cooling time for a given percentage of height loss at each imprint temperature (Fig. 6).

For instance, to limit height loss to $5 \%$ for resist patterns, cooling down after stamp removing above $T_{\mathrm{g}}$ has to be shorter than $128 \mathrm{~s}$ at $80{ }^{\circ} \mathrm{C}$ and shorter than $2 \mathrm{~s}$ at $110^{\circ} \mathrm{C}$. It is well admitted that increasing the printing temperature will reduce the resist viscosity and therefore the printing time. Nevertheless, the time needed to cool down the stamp-substrate before demolding will be longer. Both processes - printing time and cooling down - have to be taken into account for cycle time. Reducing the printing temperature would increase printing time, however, reduce cooling down. Fig. 6 allows to optimize the high demolding temperature and as a consequence the printing temperature.

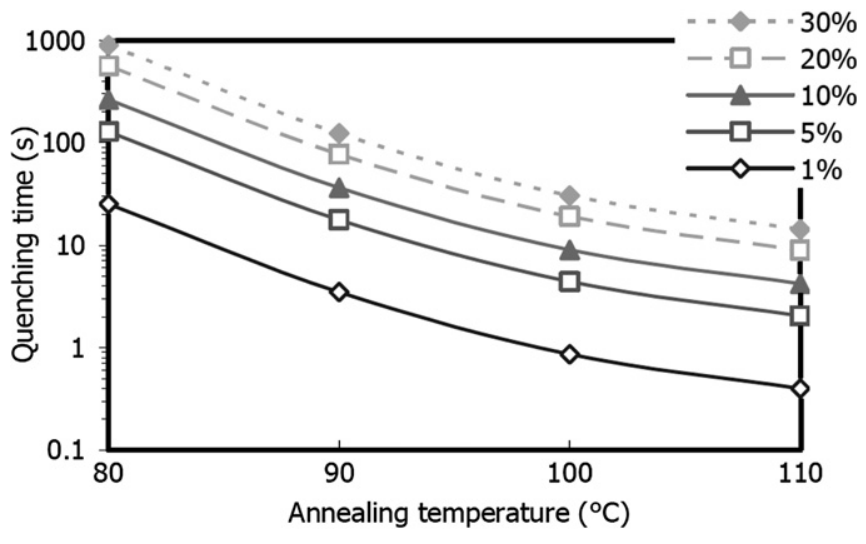

Fig. 6. Process window for quenching time versus annealing temperature. Height losses are ranging from $1 \%$ to $30 \%$.

\subsection{Mechanical analysis}

The reflow phenomenon is driven by surface free energy and slowed down by dynamic viscosity of resist. For such liquid flow [6], characteristic flow velocity, $V^{*}$, may be defined by the ratio of the driving and dissipative force.

$\frac{\sigma}{\eta} \equiv \frac{\left[\mathrm{J} \mathrm{m}^{-2}\right]}{[\mathrm{Pa} \mathrm{s}]}=\frac{\left[\mathrm{N} \mathrm{m}^{-1}\right]}{\left[\mathrm{N} \mathrm{s} \mathrm{m}^{-2}\right]}=\mathrm{m} \mathrm{s}^{-1} \equiv V^{*}$.

In our approach $V^{*}$ may be compared to height loss velocity of the resist patterns (Fig. 5). Increasing demolding temperature leads to higher reflow velocity, commensurable to $h_{0} / \tau$. On the one hand, it should be noticed that surface free energy is not supposed to vary so much between $T_{\mathrm{g}}+20{ }^{\circ} \mathrm{C}$ and $T_{\mathrm{g}}+50{ }^{\circ} \mathrm{C}$ [7]. On the other hand, reflowing velocity is almost two decades faster at $T_{\mathrm{g}}+50^{\circ} \mathrm{C}$ than at $T_{\mathrm{g}}+20^{\circ} \mathrm{C}$. It implies that temperature dependency of reflowing behavior is mainly due to temperature dependency of viscosity.

Most of efforts are now dedicated to optimize the polymer viscosity in order to reduce the printing time. Nevertheless, we demonstrated that surface free energy of the polymer should be carefully considered not only for the adhesion properties but also for the mechanical strength when the demolding is performed above $T_{\mathrm{g}}$.

\section{Conclusion}

Carry out a demolding at imprint temperature may imply a loss of pattern quality. We demonstrated that the control of quenching time of an imprint allow to control precisely pattern dimensions. Although reducing resist dynamic viscosity is known as the best way to improve imprint time, we showed that decreasing resist surface free energy will improve pattern shape, when demolding is performed at temperature above $T_{\mathrm{g}}$. Our results clearly highlighted interests for tailor-made resists with very low surface free energy, for imprint throughput improvement. 
Moreover, theoretical works are underway to clearly describe resist reflow and identify the role of polymer viscosity and surface free energy.

\section{References}

[1] S.Y. Chou, P.R. Krauss, P.J. Renstrom, Appl. Phys. Lett. 67 (1995) 3114.

[2] F. Hua, Y. Sun, A. Gaur, M. Meitl, L. Bilhaut, L. Rotkina, J. Wang, P. Geil, M. Shim, J.A. Rogers, Nano Lett. 12 (2004) 2467-2471.
[3] M. Tormen, F. Romanato, M. Altissimo, L. Businaro, P. Candeloro, E.M. Di Fabrizio, J. Vac. Sci. Tec. B 22 (2004) 767-770.

[4] H. Schift, L. Heyderman, Nanorheology - squeezed flow in hot embossing of thin films, in: C. Sotomayor (Ed.), Alternative Lithography - Unleashing the Potential of Nanotechnology, Torres of Nanostructure Science and Technology, Kluwer Academic/Plenum Publishers, 2003, pp. 46-76.

[5] C. Perret, C. Gourgon, F. Lazzarino, J. Tallal, S. Landis, R. Pelzer, Microelectron. Eng. 73-74 (2004) 172-177.

[6] P.-G. de Gennes, F. Brochard-Wyart, D. Quere, Gouttes, bulles, perles et ondes, Belin, Paris, 2005, pp. 127-137.

[7] I. Vilfan, F. Lançon et, E. Adam, Surf. Sci. 440 (1999) 279. 